\title{
MECHANICAL STABILITY STUDY OF TYPE IV CRYOMODULE (ILC PROTOTYPE)*
}

\author{
M.W. McGee $\#$, R. Doremus and C. R. Wands, \\ Fermi National Accelerator Laboratory, Batavia, IL 60510, U.S.A.
}

\begin{abstract}
An ANSYS modal and harmonic finite element analysis (FEA) was performed in order to investigate cryomodule design mechanical stability for the proposed International Linear Collider (ILC). The current cryomodule, designated Type IV or (T4CM), closely follows the Type III TESLA Test Facility (TTF) version used at DESY, with the exception of a proposed location of the superconducting (SC) quadrupole at the center. This analysis considered the stringent stability criteria established for the ILC, where vertical motion for the SC quadrupole is limited to the micron range at a few $\mathrm{Hz}$. Model validation was achieved through Type III cryomodule vibration measurement studies performed at DESY. The effect of support location, support stiffness and other important parameters were considered in a parametric sensitivity study. FEA results, fast motion investigations and stabilization techniques are discussed.
\end{abstract}

\section{INTRODUCTION}

Cryomodule development has progressed over a decade with the participation of several laboratories and institutions such as DESY, Fermilab, INFN-Milan, INFNPisa, JLab, KEK, CEA-Saclay and SLAC [1-3]. The T4CM contains eight $1.3 \mathrm{GHz}$, SC radio frequency (RF) cavities. Considered a prototype for the ILC linac, further type IV cryomodule development is necessary for optimizing column support post location, external support location and internal pipe sizes. The proposed ILC cryomodule configuration considers a 9-8-9 combination with the middle cryomodule containing a SC quadrupole. The details of design as reflected in Figures 1 and 2 in terms of inner diameter were established in late 2005 during a series of meetings held at both INFN-Milan and Fermilab.

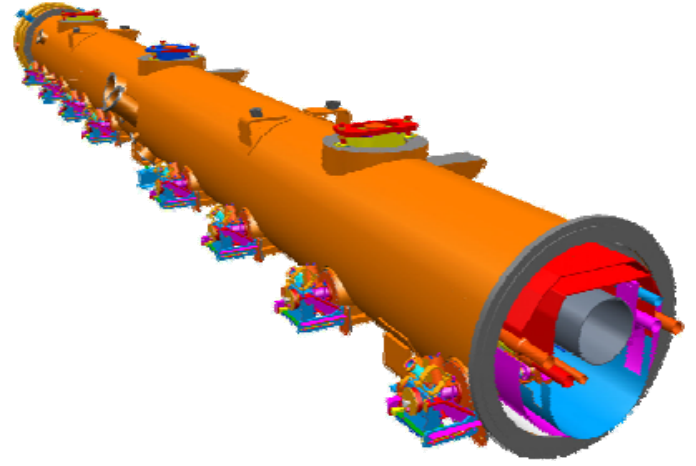

Figure 1: T4CM solid-model layout.

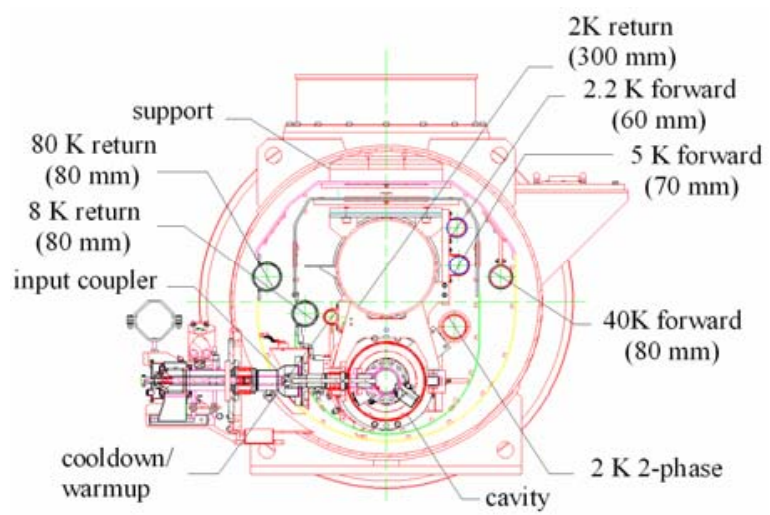

Figure 2: T4CM section view.

Improving beam stability by relocating the quadrupole to the cryomodule center is the main goal of the T4CM design. The stability criteria of $300 \mu \mathrm{m}$ was defined in 2004 [4] with a recent estimate that considers vertical quadrupole motion, limiting it to $30 \mathrm{~nm}$ in a frequency range between $10 \mathrm{~Hz}$ to $100 \mathrm{~Hz}$ [5]. Beam-based feedback through dipole corrector adjustment within the quadrupole package can correct for motion beneath 10 $\mathrm{Hz}$.

\section{FEA ANALYSIS}

A series of FEA studies in ANSYS v11 [6] were completed to compare the T4CM to the TTF cryomodule and evaluate the dynamics of the proposed T4CM design. Component Mode Synthesis (CMS) [7], an application supported by ANSYS, allows for efficient analysis of dynamic problems; breaking them up into individual superelements. Within CMS, a four part process was 
followed: a generation pass (constructing superelements), a use pass (combining superelements), an expansion pass (regeneration of superelements considering coupled solution) and the assembly view (post-generation viewing). Three type IV cryomodules in 9-8-9 configuration and TTF 8-8 (with end quad)-8 configuration with fixed end conditions were generated using CMS. External connections to ground are simply supported and allowed to slide longitudinally at the upstream (US) external support. Modeling connections was difficult since loads will exist at the interfaces where bellows are found; added stiffness was provided for these conditions.

In both designs, the cold mass hangs from three column support posts constructed from G-10 fiberglass composite, which are attached to the top of the vacuum vessel. The helium gas return pipe (HeGRP), supported by the three columns act as the coldmass spine, supporting the cavity string, quadrupole and ancillaries. Internally, connections between the HeGRP and individual cavities are allowed to slide longitudinally using a combination element (with spring constant and scaled sliding force) implemented at the coincident Mdof during the use pass phase. With the center post fully constrained, the two outboard column support posts are allowed to move longitudinally by design since thermal contraction and expansion can occur during cooldown and warmup [8]. Relative vertical and transverse alignment (or position) of the cavity string and quadrupole is held by an Invar rod (a material with very low thermal expansion) which is included within the model.

\section{Modal Studies}

Modal studies completed in ANSYS provide the fundamental mode shapes of both TTF and Type IV cryomodules and qualatively evaluate the relocation of the SC quadrupole to the center. These studies formed a spring-board for future model development. The modes described in Table 1 may be coupled (a mode that has motion contributed by two or more orthogonal directions) or completely uncoupled.

Table 1: Comparison of T4CM and TTF Modal Shapes.

\begin{tabular}{|c|c|c|c|c|}
\hline \multirow[b]{2}{*}{ Mode } & \multicolumn{2}{|c|}{ T4CM } & \multicolumn{2}{|c|}{ TTF } \\
\hline & $\begin{array}{c}f \\
(\mathbf{H z})\end{array}$ & Shape & $\begin{array}{c}f \\
(\mathrm{~Hz})\end{array}$ & Shape \\
\hline 1 & 11.0 & $\mathrm{~L}$ & 8.4 & \multirow{3}{*}{ VL } \\
\hline 2 & 12.0 & $T$ & 8.4 & \\
\hline 3 & 12.2 & \multirow[t]{2}{*}{ LQ } & 8.6 & \\
\hline 4 & 12.2 & & 9.4 & \multirow{3}{*}{ LV } \\
\hline 5 & 14.2 & \multirow{4}{*}{ LV } & 10.4 & \\
\hline 6 & 14.9 & & 10.9 & \\
\hline 7 & 14.9 & & 11.2 & $T$ \\
\hline 8 & 15.1 & & 11.3 & \multirow[t]{2}{*}{ VL } \\
\hline 9 & 15.2 & \multirow{3}{*}{ LQ } & 11.4 & \\
\hline 10 & 15.2 & & 13.2 & \multirow{3}{*}{ LV } \\
\hline 11 & 15.6 & & 13.2 & \\
\hline 12 & 15.9 & VL & 13.4 & \\
\hline 13 & 16.7 & $\mathrm{LV}$ & 14.2 & $\mathbf{T}$ \\
\hline
\end{tabular}

\begin{tabular}{|c|c|c|c|c|}
\hline 14 & 17.5 & & 15.1 & \multirow{3}{*}{ LV } \\
\hline 15 & 17.8 & \multirow[t]{2}{*}{$T$} & 15.1 & \\
\hline 16 & 17.9 & & 15.4 & \\
\hline 17 & 18.6 & \multirow{3}{*}{ LQ } & 16.1 & $\mathrm{LV}$ \\
\hline 18 & 19.4 & & 18.3 & $\bar{T}$ \\
\hline 19 & 19.5 & & 18.4 & $\mathrm{~T}$ \\
\hline 20 & 20.2 & VL & 18.6 & VL \\
\hline
\end{tabular}

Interpretation of Table 1 is as follows: primary modes are shown in red, secondary in blue, ternary in green and quandary in brown. Coldmass motion direction is given as $\mathrm{V}$ (vertical), $\mathrm{T}$ (transverse) and $\mathrm{L}$ (longitudinal) with multiple component motion described in two parts, the most significant is the first. It is also important to note cases where motion is occurring within the coldmass, yet the quadrupole is quiescent (denoted as Q). Table cells which are merged indicate multiples of a mode and these modes are symmetric. The most undesirable modal shapes are denoted in bold face font such as transverse pendulum and vertical motion. Figure 3 provides an example of extreme transverse pendulum motion found in both models.

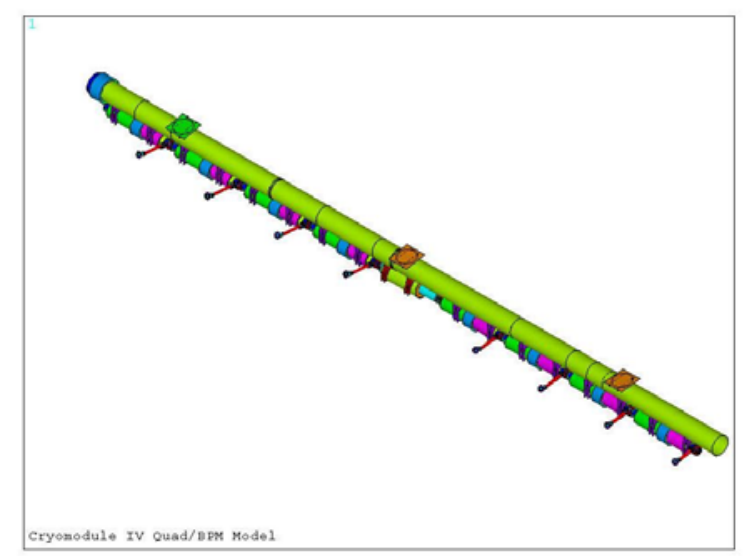

Figure 3: Transverse pendulum mode for T4CM.

Summary from Table 1 comparison:

- Mode $2(12 \mathrm{~Hz})$ of T4CM and TTF mode $7(11.2 \mathrm{~Hz})$ have identical transverse pendulum motion as the modes have shifted to higher order and their number reduced to one-half.

- Mode $12(15.9 \mathrm{~Hz})$ of T4CM and TTF modes 8 and 9 (11.3 and $11.4 \mathrm{~Hz}$ ) have similar vertical motion with a longitudinal component. Pure longitudinal motion does not present a problem for quadrupole stability. However, in these cases the longitudinal motion has a vertical component and vertical rotation.

- Modes 5, 6, 7 and 8 (14.2, 14.9, 14.9 and $15.1 \mathrm{~Hz})$ of T4CM are similar to TTF modes 4,5 and $6(9.4,10.4$ and $10.9 \mathrm{~Hz}$ ). However, the T4CM modes are higher in frequency.

- Finally, modes 3, 4, 9, 10, 11, 17, 18 and 19 have longitudinal motion with anchor points at the center quadrupole. Longitudinal momentum is imparted from 
one cavity (or cluster of cavities) to another in a series of collisions, yet the quadrupole remains quiescent.

\section{Measurement and Validation}

Devices from geophones [9-11] to wire position monitors (WPM) by Bosotti et al. [12] have provided relative displacement and frequencies given different conditions regarding the TTF cryomodule, warm and cold. From these studies and real-time measurement, extensive vibration results collected by DESY are available on-line [13].

The most important initial consideration of the FEA model involved validation. Using the existing TTF cryomodule design as our only reference, each FEA model was developed through the same process. Warm vibration measurements were taken at DESY on the TTF type III, cryomodule \#6 in 2006 [11]. Several locations were considered such as at the base (or external support), on top of the vacuum vessel, inside of the HeGRP and on top of the quadrupole. These measurements were then used in a harmonic study of the TTF cryomodule model to confirm the accuracy.

A known vertical displacement (or forcing function) taken from the DESY TTF cryomodule \#6 measurements [11] was applied to a specific node corresponding to a measurement location at a given frequency and the response found through iterative ANSYS simulation. Figure 4 depicts the measured PSD displacement at the ground on the US external support and on top of the vacuum vessel, downstream (DS). The ANSYS predicted response at the point found on top of the vacuum vessel is also plotted for comparison. Data points selected reflect a coherence between ground and vessel top signals greater than 0.8 .

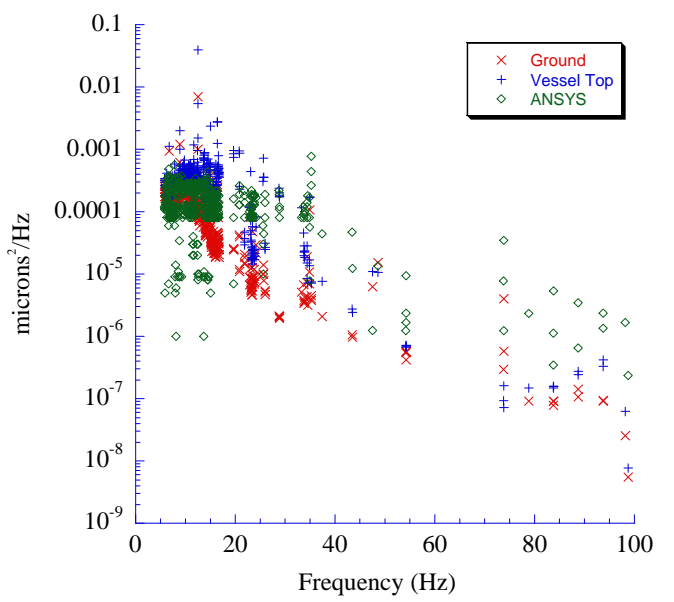

Figure 4: PSD ANSYS prediction of vertical response.

\section{Sensitivity Studies}

Sensitivity studies will impact future cryomodule design as parameters for T4CM have been defined for procurement reasons. Observing significant changes in model response to slight parametric adjustment is a crucial tool of this FEA study and is ultimately the goal. These studies are under way with updated modal, harmonic and sensitivity studies provided on-line.

\section{FUTURE WORK}

Sensitivity studies continue for next generation cryomodule design. Three cryomodules (two TTF style cryomodules and a T4CM connected in series) will be tested in New Muon Laboratory (NML) at Fermilab. During these tests, geophones will measure technical and cultural noise while operating at $1.8 \mathrm{~K}$ using techniques developed at DESY [12].

Currently, TTF style cryomodules are designed to hang from the enclosure ceiling. The T4CM plan involves supporting from the floor. Measurements are planned at DESY involving these hanging supports to evaluate. External support design investigation is necessary to ensure mechanical stability and offer isolation from outside noise sources by design. FEA studies are also planned, first considering the hanging support TTF configuration at DESY then evaluating the TTF and T4CM floor support design at NML, Fermilab.

\section{ACKNOWLEDGEMENTS}

Special thanks to Ingrid Fang, Ang Lee and Zhang Tang of PPD/Analysis Group at Fermilab for their computational and ANSYS related assistance. Also, thanks to Ramila Amirikas and Alessandro Bertolini for their essential help regarding model validation. Thanks to Harry Carter, Don Mitchell, Tom Nicol and Tom Peterson of Fermilab for their support regarding the T4CM design team. Special thanks to Stewart Mitchell for his constant computer support.

\section{REFERENCES}

[1] D.A. Edwards, "TESLA Test Facility Linac Design Report”, DESY Print, March 1995, TESLA 95-01.

[2] TESLA Technical Design Report, Part II: The DESY 2001-011, ECFA Accelerator, March 2001, D2001209, TESLA Report 2001-23, TESLA-FEL 2001-05.

[3] C. Pagani et al., "Construction, Commissioning and A Test Cryogenic Performances of the First TESLA Facility (TTF) Cryomodule”, Advances in Cryogenic Engineering, Vol 43, 1998, p. 87.

[4] N. Walker, "Beam Dynamics Tolerances for Module Design”, DESY/FNAL Module Meeting at DESY, June 2004.

[5] N. Solyak, "Vibration Requirements", ILC Cryomodule Instrumentation Meeting at FNAL, March 2007.

[6] http://www.ansys.com

[7] U. Sellgren, "COMPONENT MODE SYNTHESIS A method for efficient dynamic simulation of complex technical systems", VISP WP5 Document Data, Stockholm, 2003.

[8] C. Pagani and P. Pierini, "Cryomodule Design, Assembly and Alignment”, SUP’04, 2004.

[9] W. Bialowons and H. Ehrlichmann, "First Results Of the Vibration Measurements with Seismic Sensors 
Atm TTF Modules”, XFEL LINAC Module Meeting Hamburg, September 2, 2004.

[10] A. Bosotti et al., "Mechanical Vibration Measurements on TTF Cryomodules”, EPAC'06, July 2006.

[11] R. Amirikas, A. Bertolini and W. Bialowons, "Vibration Stability Studies of a Superconducting Accelerating Module at Room Temperature", PAC'07, also in these proceedings, 2007.

[12] R. Amirikas, A. Bertolini and W. Bialowons, "Vibration Stability Studies of a Superconducting Module at 2K”, PAC'07, also in these proceedings, 2007.

[13] http://ground-vibrations.desy.de 